\title{
Construction of Reference Data for Classification of Elasticity Images of Arterial Wall
}

\author{
Jun Inagaki, Hideyuki Hasegawa and Hiroshi Kanai \\ Graduate School of Engineering, Tohoku University, \\ Sendai 980-8579, Japan \\ Email: inagaki@us.ecei.tohoku.ac.jp
}

\author{
Masataka Ichiki \\ Sendai Hospital of \\ East Railway Company, \\ Sendai, Japan
}

\author{
Fumiaki Tezuka \\ Sendai Medical Center, \\ Sendai, Japan
}

\begin{abstract}
We have developed the phased tracking method [1] for measuring the minute change in thickness during one heartbeat and the elasticity of the arterial wall. By comparing the pathological findings with the elasticity distribution measured with ultrasound, statistic parameters for respective tissues in the arterial wall were determined. We have already measured the statistic parameters for lipid and a mixture of smooth muscle and collagen fiber [2]. In this paper, elasticity distributions were measured for blood clot and calcified tissue. Then, we discussed whether the measured elasticity distributions can be used as reference data for classifying cross-sectional elasticity images. In addition to the measurement of elasticity distribution, the correlation between the collagen content and the elasticity was investigated for fibrous tissue. Collagen is supposed to mainly contribute to the stability of the fibrous cap of atherosclerotic plaque. Therefore, such a correlation between elasticity and a tissue element of the wall may provide useful information on diagnosis of plaque vulnerability.
\end{abstract}

\section{INTRODUCTION}

Cerebral infarction and myocardial infarction are mainly caused as terminal symptoms of atherosclerosis. Therefore, it is important to diagnose atherosclerosis in an early stage. Rupture of atherosclerotic plaque is probably the most important factor underlying the sudden outbreak of the acute coronary syndrome [3]. Direct characterization of the composition and vulnerability of atherosclerotic plaque may offer insight into the mechanism of plaque regression and progression [4], [5]. The change in elasticity is caused greatly by the change in composition of the arterial wall due to development of atherosclerosis [6]. Therefore, the measurement of elasticity has potential for tissue characterization of plaque. In this paper, the reference data of elasticity distributions for respective tissues were constructed to classify arterial tissues from the elasticity images measured with transcutaneous ultrasound. From in vitro experiments using extracted human femoral arteries, the relation between tissue components in the arterial wall and the elasticity was investigated by comparing elasticity images measured by ultrasound with the corresponding pathological images.

\section{METHODS}

\section{A. Elasticity Estimation}

An ultrasonic beam was sequentially scanned at $M(=60)$ positions with a linear-type ultrasonic probe of $7.5 \mathrm{MHz}$ using conventional ultrasound diagnostic equipment, and multiple
$\left(N_{m}+1\right)$ points were preset from the luminal surface to the adventitia along the $m$-th ultrasonic beam $(m=1, \ldots, M)$ with constant intervals of $75 \mu \mathrm{m}$ at a time $t_{0}$ just before the ejection period. By dividing the arterial wall into multiple layers, we defined the $n$-th layer $\left(n=1, \ldots, N_{m}\right)$ as being between two contiguous points, $n$ and $n+1$, along each beam. For measurement of change in thickness of each of the $N_{m}$ layers, the instantaneous depth $x_{m, n}(t)$ of the $n$-th point along the $m$-th beam was simultaneously tracked by applying the phased tracking method [1] to the received ultrasound. The minute decrease of several tens of a micrometer in thickness of the $n$-th layer resulting from the arrival of the pressure wave at the beginning of the ejection period was determined as $\Delta h_{m, n}$.

In the phased tracking method, for calculation of the auto correlation function between the quadrature demodulated signals of sequentially received echoes, minute phase change of about 0.4 degrees caused by movement of the $n$-th point during the pulse transmission interval $\Delta T(=200 \mu \mathrm{s})$ can be accurately determined by introducing a constraint, namely, that their waveforms are identical but their phase values change [1], [7]. The lowest value of the change in thickness was validated as being about $0.5 \mu \mathrm{m}$ by expanding a rubber plate in a water tank [8]. Such a minute change in thickness cannot be measured by any other method. This method has already been applied to the in vivo detection of regional instantaneous displacement and change in thickness, with sufficient reproducibility, in the carotid arteries [9].

From the ratio of the maximum decrease in thickness during one heartbeat, $\Delta h_{m, n, \max }=\max _{t}\left|\Delta h_{m, n}(t)\right|$, to the initial thickness, $h_{0}$, of the $n$-th layer, the maximum deformation of the $n$-th layer was obtained by $\Delta \varepsilon_{m, n, \max }=\Delta h_{m, n, \max } / h_{0}$. Since the deformation was sufficiently small and was in the linear regime, it showed incremental strain in the radial direction. By assuming that the arterial wall is incompressible and that the blood pressure is applied normal to each layer, the elastic modulus of the $n$-th layer along the $m$-th beam, $E_{\theta, m, n}$, is approximately given by [10]

$$
E_{\theta, m, n} \approx \frac{1}{2}\left(\frac{\rho_{m, n, 0}}{h_{0} \cdot N_{m}}+\frac{N_{m}-n+1}{N_{m}}\right) \frac{\Delta p}{\left|\Delta \varepsilon_{m, n, \max }\right|},
$$

where $\rho_{m, n, 0}$ is the initial inner radius of curvature of the $n$ th layer along the $m$-th beam at a time $t_{0}$. We assumed that 
the pressure in the arterial wall decreases linearly with the distance from the intimal side to the adventitia and that the arterial wall is almost isotropic [11].

\section{B. System for in vitro experiments}

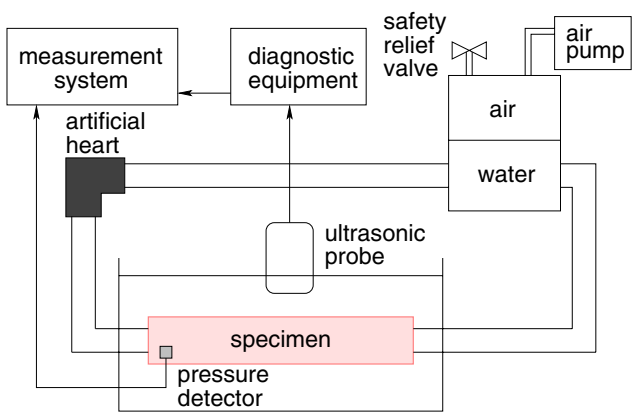

Fig. 1. Schematic diagram of system for in vitro experiments.

Figure 1 shows the experimental setup for in vitro experiments using extracted human femoral arteries. The change in internal pressure was generated using an artificial heart, and the internal pressure was measured by the pressure detector placed in lumen of the artery. The artery fixed in the water tank was measured in the long-axis plane with a linear-type probe (center frequency: 7.5 MHz).

\section{Construction of Elasticity Distribution for Each Tissue}

In this paper, the elasticity distributions were obtained further with respect to blood clot and calcified tissue. After ultrasonic elasticity measurements, the arteries were fixed in formalin. The plane scanned by ultrasound was identified by imaging the needle, which was fixed in the external surface of the posterior wall, in the B-mode image during the ultrasonic measurement [2]. The pathological images of the measured sections were made with Elastica-Masson staining for blood clot and with Hematoxylin-Eosin staining for calcified tissue. By referring the pathological images, the regions which correspond to blood clot and calcified tissue were assigned in the cross-sectional elasticity images. From the assigned regions for respective tissues, the elasticity distributions were determined.

\section{Collagen Content Estimation}

Collagen is considered to mainly contribute to the stiffness of the fibrous cap of plaque. Thus, the correlation between the collagen content and the elasticity was investigated.

In the elasticity image, there are $M$ ultrasonic beams and $N_{m}$ regions along $m$-th beam $(m=1, \ldots, M)$. Therefore, in the elasticity image, there are $\sum_{m=1}^{M} N_{m}$ regions with respective elasticity values. As shown in Figure 2, each region in the elasticity image can be compared with the corresponding region in the pathological image by dividing the entire pathological image, which corresponds to the entire elasticity image, into $M$ sections in the axial direction of the artery and dividing each $m$-th section of $M$ sections into $N_{m}$ regions in the radial direction. The sizes of all regions in the elasticity image are same. However, sizes of regions assigned in the pathological image become different because of the distortion of the arterial wall due to dehydration during formalin fixation. In this paper, the change in size due to distortion is assumed to be homogeneous.

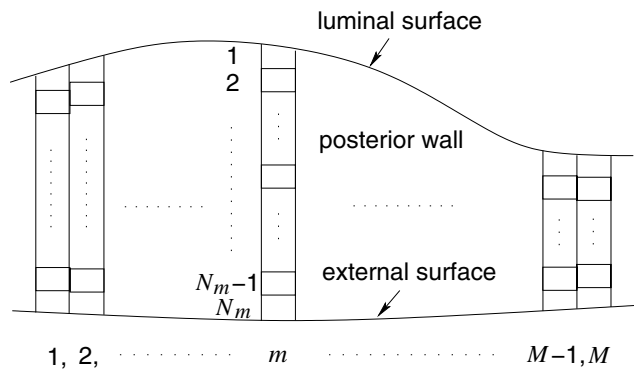

Fig. 2. Illustration of assignment of regions.

Then, the collagen content of each region assigned in the pathological image was estimated using the Mahalanobis distance. In this paper, the composition of each region were classified into 4 classes ( $i=1$ : collagen, 2: smooth muscle, 3: elastin, 4: background). The mean, $\boldsymbol{\mu}_{i}=\left(\mu_{r}, \mu_{g}, \mu_{b}\right)^{T}$, and covariance matrix, $\sum_{i}$, of RGB values were determined for each class, $i$, by assigning a typical area for each class in the pathological image. From the determined mean, $\boldsymbol{\mu}_{\boldsymbol{i}}$, and the covariance matrix, $\sum_{i}$, the Mahalanobis distance, $d_{i}^{2}$, between each pixel, $\boldsymbol{X}=\left(x_{r}, x_{g}, x_{b}\right)^{T}$, and the center of $i$-th class can be obtained as follows:

$$
d_{i}^{2}=\left(\boldsymbol{X}-\boldsymbol{\mu}_{i}\right)^{T} \sum_{i}^{-1}\left(\boldsymbol{X}-\boldsymbol{\mu}_{i}\right) .
$$

The pixel, $\boldsymbol{X}$, is classified into class $i_{\min }$ with the least Mahalanobis distance to $\boldsymbol{X}$.

To evaluate the direct contribution of the collagen content to the elasticity, we employed the artery mainly consists of fibrous tissue. There are no blood clot and calcified tissue.

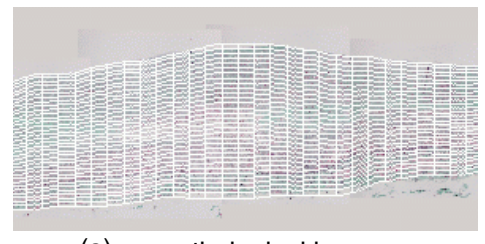

(a) pathological image

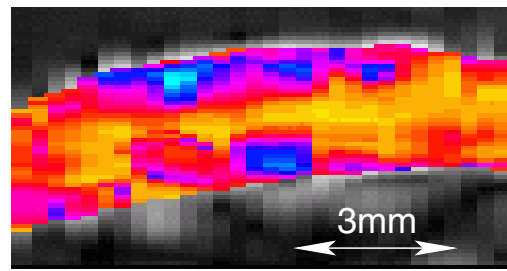

(b) elasticity image

Fig. 3. (a) Pathological image divided into each region. (b) Elasticity image corresponds to the section of (a).

Figure 3(a) shows the pathological image divided into regions which correspond to those in the elasticity image. Figure 3(b) shows the elasticity image of the section same as the pathological image. In the process for creating the 
pathological image, some deformations occur unavoidably. Such a deformation leads difference in size and shape between the elasticity and pathological image. Therefore, we employed a part of the arterial wall with little difference in size and shape between the elasticity image and pathological image.

The spatial resolution of the elasticity image was $300 \mu \mathrm{m}$ by $75 \mu \mathrm{m}$ in the axial and radial directions [2]. This resolution size was too small comparing with the deformation caused by dehydration, therefore, we employed the spatially averaged values of 2 (axial direction) $\times 8$ (radial direction) regions in each image. Thus, the averaged size is $600 \mu \mathrm{m}$ in axial direction and $600 \mu \mathrm{m}$ in radial direction.

\section{RESULTS}

\section{A. Construction of Elasticity Distribution for Each Tissue}

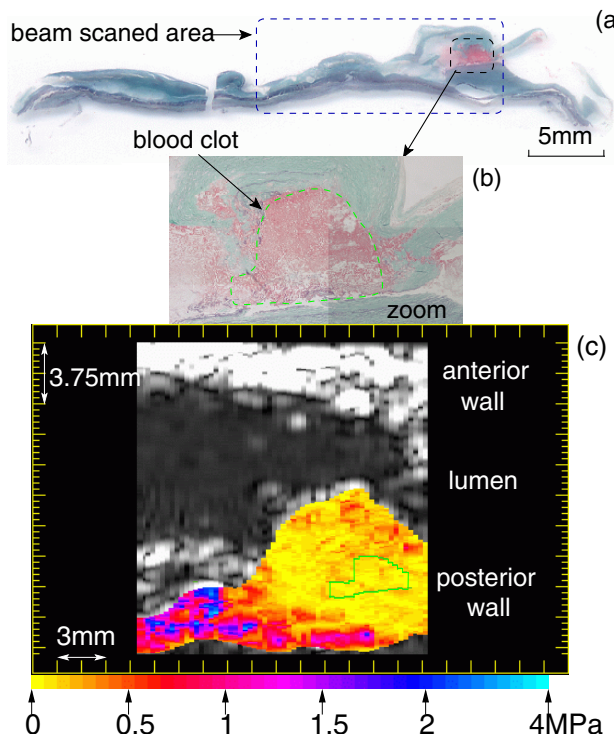

(a)

(c)

Fig. 4. (a) Pathological image of the posterior wall of an extracted human femoral artery made with Elastica-Masson staining. (b) Enlarged view of the blood clot shown in (a). (c) Elasticity image.

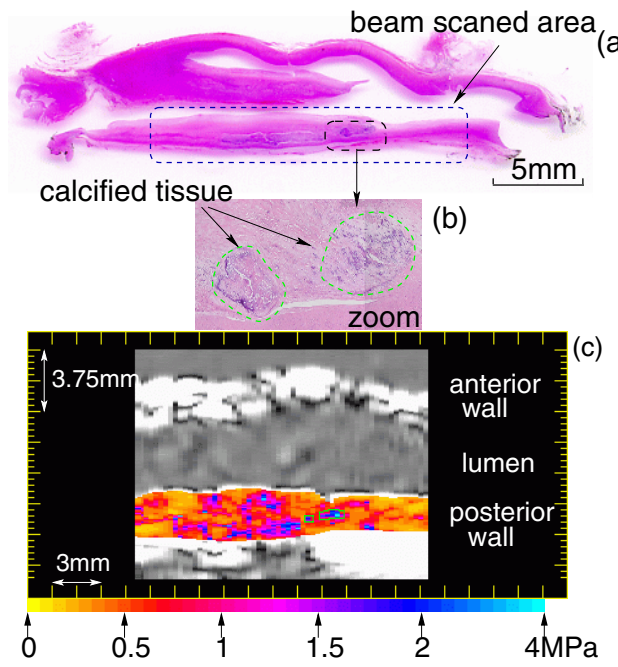

Fig. 5. (a) Pathological image of the posterior wall of an extracted human femoral artery made with Hematoxylin-Eosin staining. (b) Enlarged view of the calcified shown in (a). (c) Elasticity image.
Figures 4(c) and 5(c) show the elasticity images of two extracted human femoral arteries. After ultrasonic measurement, the pathological images of the measured section were made as shown in Figs. 4(a) and 5(a) with Elastica-Masson staining and Hematoxylin-Eosin staining, respectively. From the respective pathological images, blood clot and calcified tissue were identified. The means and the standard deviations in elasticity were determined to be $96 \pm 57 \mathrm{kPa}$ for blood clot and $1.4 \pm 0.68 \mathrm{MPa}$ for calcified tissue from the number of points of 612 and 218 assigned in the elasticity image.
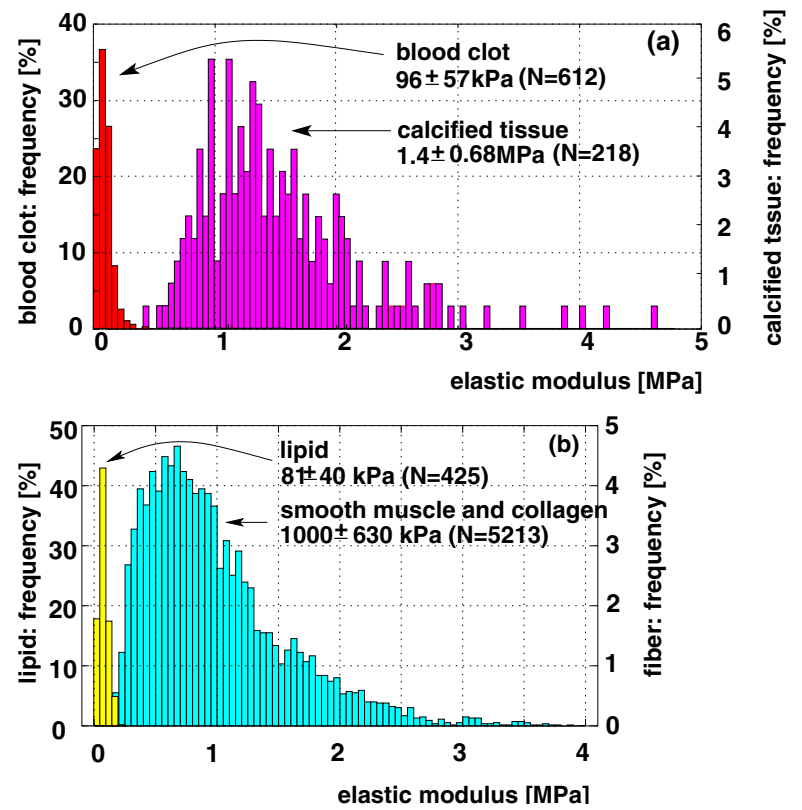

Fig. 6. (a) Elasticity distributions of blood clot and calcified tissue. (b) Elasticity distributions of lipid and mixture of smooth muscle and collagen fiber [2].

Figure 6 shows the elasticity distributions of blood clot, calcified tissue, lipid, and fibrous tissue (mixture of smooth muscle and collagen fiber) [2]. By comparing each elasticity distribution, the high similarity was found in the elasticity distributions of lipid and blood clot. It means that the classification of these tissues based on elasticity is difficult. Moreover, the elasticity distributions of fibrous tissue and calcified tissue largely overlapped each other. However, fortunately, it will be possible to classify lipid or blood clot from fibrous tissue or calcified tissue. These two groups (soft and hard tissues) are separated at about $300 \mathrm{kPa}$. These results suggest that hard tissue such as fibrous tissue and calcified tissue, which contributes to stability of plaque, can be detected based on elasticity.

\section{B. Collagen Content Estimation}

Figure 7 shows the correlation between the ratio (collagen content) of the area, which was classified into collagen, and the elasticity. In Fig. 7, one plot was obtained by spatial averaging with the size of $600 \mu \mathrm{m} \times 600 \mu \mathrm{m}$. The positive correlation between the elasticity and the collagen content was found. 


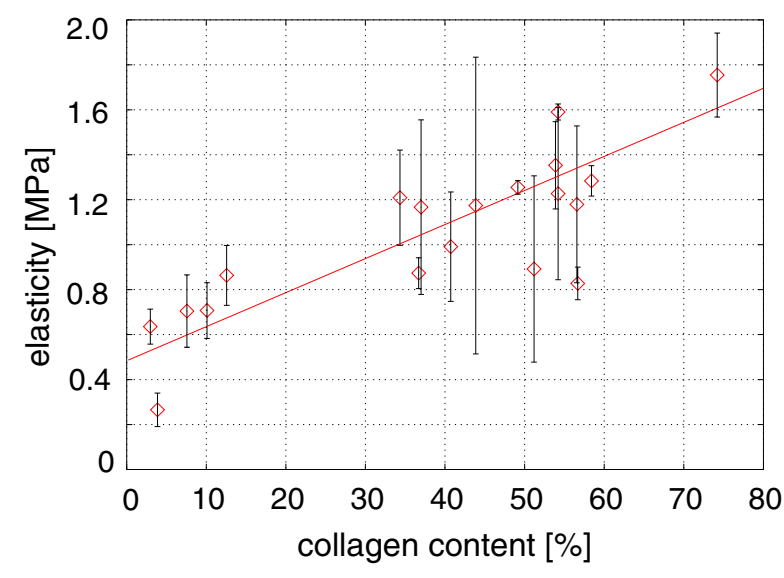

Fig. 7. Relation between the collagen content and the elasticity.
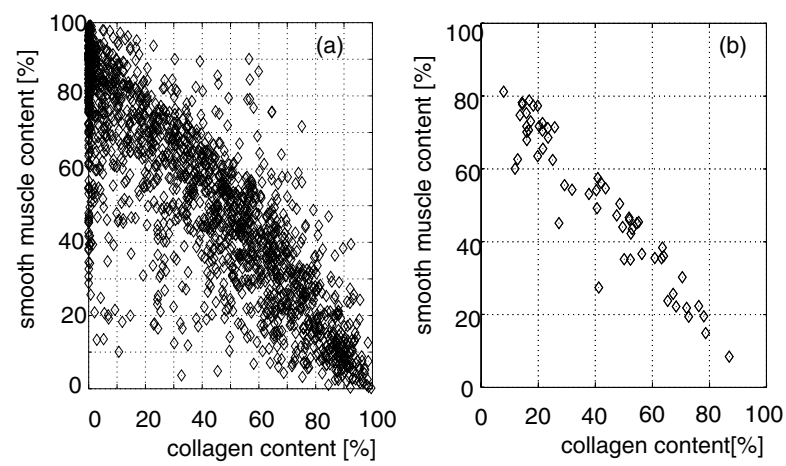

Fig. 8. Relation between collagen content and smooth muscle content. (a) The correlation before spatial averaging. (b) The correlation after spatial averaging.

Figures 8(a) and 8(b) show the relation between the collagen content and the smooth muscle content before and after spatial averaging. There were some points that the sum of both contents was over 100\% in Fig. 8(a). In Figs. 8(a) and 8(b), region assignments were manually performed two times for estimation collagen and smooth muscle contents. Because the size of each assigned region is small and the size of the wall is changed by formalin fixation, it is difficult to identify a region in the elasticity image as the corresponding region in the pathological image. Spatial averaging reduces the difficulties in the region identification. As shown in Fig. 8(b), all the sums of collagen and smooth muscle content becomes below $100 \%$ after spatial averaging. This result suggests that the influence of region disagreement was reduced. In Fig. 8(b), the sums of both contents mostly showed about $90 \%$. The correlation between the smooth muscle content and the elasticity will be negative. The correlation between the collagen content and the elasticity was superimposed on the elasticity distribution in Fig. 6(b) of fibrous tissue as shown in Fig. 9. It is found from Fig. 9 that the large elasticity components consist of collagen.

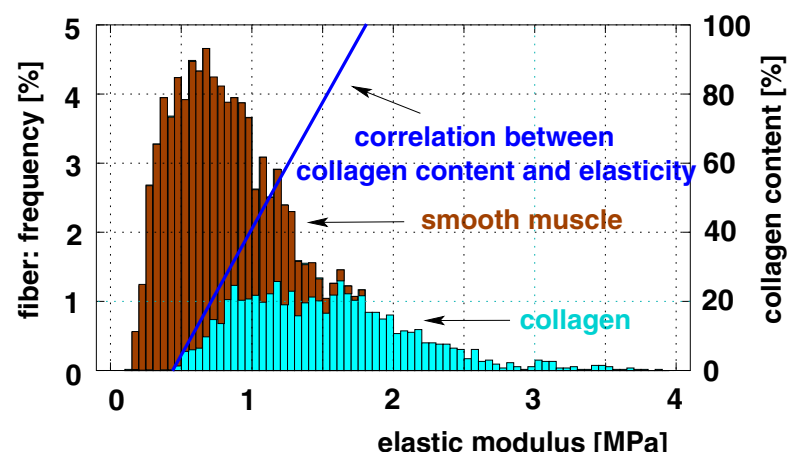

Fig. 9. Elasticity distribution for fibrous tissue divided into collagen and smooth muscle.

\section{CONCLUSION}

In this paper, the elasticity distributions for blood clot and calcified tissue were measured with ultrasound. The comparison of the elasticity distributions including that for lipid and fibrous tissue [2], suggest the possibility of classifying the arterial tissues as soft tissues (lipid and blood clot) or hard tissues (fibrous tissue and calcified tissue) from the elasticity image. These two groups are separated at about $300 \mathrm{kPa}$, this elastic modulus may be important to make a rough estimate of the tissue characterization based on elasticity. Furthermore, the positive correlation between elasticity and collagen content was found in fibrous tissue. It offers the potential for estimation of the collagen content by the elasticity and contributes for the evaluating vulnerability of the atherosclerotic plaque.

\section{REFERENCES}

[1] H. Kanai, M. Sato, Y. Koiwa, and N. Chubachi, "Transcutaneous Measurement and Spectrum Analysis of Heart Wall Vibrations," IEEE Trans. Ultrason. Ferroelect. Freq. Contr., Vol. 44, No. 2, pp. 791-810, 1996.

[2] H. Kanai, H. Hasegawa, M. Ichiki, F. Tezuka, and Y. Koiwa, "Elasticity Imaging of Atheroma with Transcutaneous Ultrasound," Circulation, Vol. 107, pp. 3018-3021, 2003.

[3] P. R. Moreno, E. Falk, I. F. Palacios, J. B. Newell, V. Fuster, and J. T. Fallon,"Macrophage Infiltration in Acute Coronary Syndromes. Implication for Plaque Rupture," Circulation, Vol. 90, pp. 775-778, 1994

[4] H. M. Loree, R. D. Kamm, R. G. Stringfellow, and R. T. Lee, "Effects of Fibrous Cap Thickness on Peak Circumferential Stress in Model Atherosclerotic Vessels," Circ. Res., Vol. 71, pp. 850-858, 1992.

[5] E. Falk, K. Prediman, P. K. Shah, and V. Fuster, "Coronary Plaque Disruption," Circulation, Vol. 92, pp. 657-671, 1995.

[6] R. T. Lee, A. J. Grodzinsky, E. H. Frank, R. D. Kamm, and F. J. Schoen, "Structure-Dependent Dynamic Mechanical Behavior of Fibrous Caps from Human Atherosclerotic Plaques," Circulation, Vol. 83, pp. 17641777, 1991.

[7] H. Kanai, H. Hasegawa, N. Chubachi, Y. Koiwa, and M. Tanaka, "Noninvasive Evaluation of Local Myocardial Thickening and Its Colorcoded Imaging," IEEE Trans. Ultrason. Ferroelect. Freq. Contr. , Vol. 44, pp. 752-768, 1997

[8] H. Kanai, K. Sugimura, Y. Koiwa, and Y. Tsukahara, "Accuracy Evaluation in Ultrasonic-based Measurement of Microscopic Change in Thickness," Electron. Lett., Vol. 35, pp. 949-950, 1999.

[9] H. Kanai, Y. Koiwa, and J. Zhang, "Real-time Measurements of Local Myocardium Motion and Arterial Wall Thickening," IEEE Trans. Ultrason. Ferroelect. Freq. Contr., Vol. 46, pp. 1229-1241,1999.

[10] H. Hasegawa, H. Kanai, N. Hoshimiya, and Y. Koiwa, "Evaluating the Regional Elastic Modulus of a Cylindrical Shell with Nonuniform Wall Thickness," J. Med. Ultrason., Vol. 31, pp. 81-90, 2004.

[11] D. J. Patel, J. S. Janicki, R. N. Vaishnav, and J. T. Young, "Dynamic Anisotropic Viscoelastic Properties of the Aorta in Living Dogs," Circ. Res., Vol. 32, pp. 93-107, 1973. 